Received Date : 10-Aug-2016

Revised Date : 19-Sep-2016

Accepted Date : 21-Sep-2016

Article type : Original Article

\title{
Multifaceted role of hair follicle dermal cells in bioengineered skins
}

\author{
C.A. Higgins ${ }^{1,2}$, M. Roger ${ }^{3}$, R. Hill ${ }^{3}$, A.S. Ali-Khan ${ }^{4}$, J. Garlick ${ }^{5}$, A.M. Christiano ${ }^{1,6}$, C.A.B. \\ Jahoda $^{3}$
}

\section{Affiliations}

1. Department of Dermatology, Columbia University, New York, NY, USA

2. Department of Bioengineering, Imperial College London, UK

3. School of Biological and Biomedical Sciences, Durham University, Durham, UK

4. Department of Plastic Surgery, University Hospital of Durham, Durham, UK

5. Sackler Graduate School of Biomedical Sciences, Tufts University, Boston, USA

6. Department of Genetics and Development, Columbia University, New York, NY, USA

\section{Corresponding author:}

Colin A.B. Jahoda

School of Biological and Biomedical Sciences,

South Road, Durham, DH1 3LE, UK

Phone: +441913341239

Fax: +441913341201

Email: colin.jahoda@durham.ac.uk

Running title: Hair follicle cells support epidermal differentiation

This article has been accepted for publication and undergone full peer review but has not been through the copyediting, typesetting, pagination and proofreading process, which may lead to differences between this version and the Version of Record. Please cite this article as doi: 10.1111/bjd.15087

This article is protected by copyright. All rights reserved. 


\section{Bulleted statements:}

Engineered skin constructs have been used in research, and clinically for three decades. This manuscript demonstrates that cells isolated from the hair follicle may have excellent capabilities for establishing engineered skins.

Funding statement: This work was supported by the Dermatology Foundation, the MRC (G1000846), the British Skin Foundation, and by the NIH/NIAMS (P20AR044535).

Disclosure: The authors have no conflicts of interest.

\section{Summary (Abstract)}

Background: The method to generate bioengineered skin constructs was pioneered several decades ago, and nowadays these constructs are used regularly for the treatment of severe burns and non-healing wounds. Commonly, these constructs are comprised of skin fibroblasts within a collagen scaffold, forming the skin dermis, and stratified keratinocytes overlying this, forming the skin epidermis. In the past decade there has been a surge of interest in bioengineered skins, with researchers searching for alternative cell sources, or scaffolds, from which constructs can be established, and for more biomimetic equivalents with skin appendages.

Objectives: In this manuscript we wanted to evaluate whether human hair follicle dermal cells can act as an alternative cell source for engineering the dermal component of engineered skin constructs.

Methods: We established in vitro skin constructs by incorporating into the collagenous dermal compartment either primary interfollicular dermal fibroblasts, hair follicle dermal papilla, or hair follicle dermal sheath cells. In vivo skins were established by mixing dermal cells and keratinocytes in chambers on top of immunologically compromised mice.

This article is protected by copyright. All rights reserved. 
Results: All fibroblast subtypes were capable of supporting growth of overlying epithelial cells, both in vitro and in vivo. However, we found hair follicle dermal sheath cells to be superior to fibroblasts in their capacity to influence the establishment of a basal lamina.

Conclusions: Human hair follicle dermal cells can be readily interchanged with interfollicular fibroblasts, and used as an alternative cell source for establishing the dermal component of engineered skin both in vitro and in vivo.

Keywords: Engineered skin, hair follicle, dermal support, basement membrane, wound healing.

\section{Introduction}

In its simplest form, human skin is essentially comprised of two layers, an outer layer of ectoderm (multilayered epidermis), and an inner layer of mesenchyme (dermis). However, the integument is actually strikingly complex, being both richly innervated and vascularized, in addition to housing a complex immune system. It is also populated with hair follicles, sebaceous, eccrine and apocrine glands, all of which arise from skin cells during development. Indeed, the skin has a range of functions additional to its primary role, which is that of a covering, or barrier ${ }^{1}$. It is the outer layer of the epidermis, the stratum corneum that provides this barrier function. This barrier not only acts as an immune defense to external pathogens, but also maintains homeostasis of water and electrolytes within the body.

When the skin barrier function is compromised, after a wound or a burn, there are four consecutive but overlapping phases to wound repair; hemostasis, inflammation, proliferation, and remodeling, modulated by synergistic interactions between the dermis and epidermis. However, there are many instances where wounds do not heal after injury, for example, in the

This article is protected by copyright. All rights reserved. 
case of diabetic or chronic venous non-healing ulcers ${ }^{2}$. Often this is as a result of one of the four stages of the healing process going awry ${ }^{3}$. These non-healing wounds account for $2-4 \%$ of the health care budget in industrialized counties, with $1 \%$ of the population affected by such a wound at any time ${ }^{4}$, and as such, development of effective treatments is a major unmet medical need. The current gold standard treatment for a full thickness wound such as a burn is a split thickness autograft, harvested from a healthy, uninjured donor site on the patient ${ }^{5}$. However, many people with diabetic ulcers, or other chronic wounds have underlying healing defects, and so autografts are not a suitable option. Moreover, there is a small subset of patients with congenital diseases that cause aberrant wound healing, or blistering of the skin, where again, autografts are not a viable treatment option ${ }^{6}$. Grafting of bioengineered skin substitutes is currently an alternative treatment, when autografts are not optimal.

Over the last three decades, development of bioengineered skin substitutes has rapidly advanced and using bioengineered skin to treat chronic wounds is now a reality ${ }^{7}$. Nevertheless, progress is still limited as no bioengineered skin can completely replicate the anatomy and physiology of an autograft ${ }^{8}$. In the 1970's Rheinwald and Green developed a method to isolate and culture keratinocytes from the epidermis ${ }^{9}$, and this initial discovery promoted the development of skin substitutes such as EpiCel $\circledast$ that provide an epidermal covering to a wound, acting as a temporary barrier replacement while promoting skin repair ${ }^{10}$. Factors present within the dermal component of the wound bed are crucial for recovery from full thickness wounds, and commercial products such as Transcyte $\AA$, or Dermagraft $₫$ have been developed that replace the dermis, providing essential growth factors and extracellular matrix to the wound bed ${ }^{8}$. There are limited bioengineered skin products that replace both the epidermis and dermal layers of the skin although Apligraf $₫$ is one such FDA approved product, composed of neonate-derived fibroblasts cultured in a bovine collagen matrix, over which neonate-derived keratinocytes are seeded to produce a differentiated epidermis ${ }^{11}$. There are now detailed

This article is protected by copyright. All rights reserved. 
protocols on composite skin establishment ${ }^{12,13}$, and an expanding network of researchers are able to establish skin models within their laboratories.

Additionally, efforts have focused on replacing the animal derived dermal matrix ${ }^{14}$, or incorporating vascular networks into the dermal tissue to promote anastomosis, which would improve survival chances of the grafted tissue ${ }^{15,16}$. Several epidermal and dermal populations can be isolated from the skin for use in skin regeneration ${ }^{17}$. However, in an effort to improve on the current bioengineered skin models, other researchers have sought to identify alternate tissue sources, for isolation of stem cells and generation of autologous skin substitutes. These include mesenchymal stem cells ${ }^{18}$, adipose cells ${ }^{16,19}$, and amniotic mesenchyme ${ }^{20}$. Another strategy has been to refine the subpopulation of skin fibroblasts being used, for example by focusing on those derived from the upper papillary layer of the dermis ${ }^{21}$ or by using fibroblasts derived from human embryonic stem cells ${ }^{22}$.

An alternative fibroblast cell source for use in skin constructs is hair follicle dermal cells, which are specialized fibroblasts. There are two populations of fibroblasts in the follicle, the dermal papilla and dermal sheath. Arising from the dermal condensate, papilla and sheath are derived from the same intermediate progenitors as papillary fibroblasts ${ }^{23}$. When isolated from the follicle and expanded by growth in culture, hair follicle dermal cells display multipotency and plasticity, and can differentiate down several mesenchymal lineages ${ }^{24}$. Moreover, in the presence of TGF- $\beta 1$ (which is expressed at high levels in wounded skin), rat dermal papilla cells transform and become more fibroblast-like ${ }^{25,26}$. We have previously hypothesized that hair follicle dermal cells have an alternate function to their role within the hair follicle (which is to promote hair growth), and can also act in a wound healing capacity in the skin ${ }^{27}$. Additionally, the presence of hair follicles within skin has long been theorized to promote healing, as rodent skin heals faster after injury when follicles are in an active growth phase, rather than their resting phase ${ }^{28,29}$. Observations on recovery times after full thickness wounds in hair bearing This article is protected by copyright. All rights reserved. 
mammals indicates that animals with higher hair densities heal faster with less scar formation, while grafting of hair follicles into chronic leg ulcers has been shown to promote wound closure 30. With these clinical observations the hair follicle disappears while promoting wound closure, suggesting that the cells are incorporated into the wound ${ }^{31}$. It addition to providing a source of cells for re-epithelialization, it is likely that the hair follicle also promotes dermal remodeling, aiding wound closure ${ }^{32}$. This remodeling may be in the form of matrix deposition, or perhaps the follicle may promote neovascularization. After all, VEGF is an angiogenic factor expressed at highest levels within dermal cells of the follicle ${ }^{33}$, while in vitro dermal papilla cells promote significantly more tubulogenesis of human microvascular endothelial cells compared to nonfollicle fibroblasts ${ }^{34}$.

We recently demonstrated that human hair follicle dermal cells act as a superior support for human keratinocytes in monolayer cultures, compared to human fibroblasts or irradiated 3T3 cells ${ }^{35}$. Additionally, hair follicle dermal papilla cells incorporated into a dermal matrix are capable of promoting contraction in skin wounds in nude mice ${ }^{36}$. We hypothesize that incorporation of hair follicle dermal cells into skin constructs used for wound covering will result in an improved bioengineered skin, as the dermal cells will act in a supporting role for the overlying keratinocytes, in addition to promoting integration, and vascularization at the wound site. Previously, hair follicle outer root sheath (epithelial), or bulge stem cells have been incorporated into skin constructs ${ }^{37-39}$, and even form the basis of bioengineered epithelial sheets that have been used for clinical application ${ }^{40}$. However, hair follicle dermal cells are an untapped source of cells for use in living bioengineered skins, and their use is potentially a stepping stone to establishing hair follicles within bioengineered skin ${ }^{41}$. Indeed recent studies have shown that human dermal papilla cells are capable of inducing hair follicles in skin constructs 42. Uniquely, hair follicle dermal cells possess immunosuppressive properties, protecting them from rejection after transplantation ${ }^{43}$. This property alone confers an active benefit over other

This article is protected by copyright. All rights reserved. 
bioengineered skins, as it opens up the possibility of utilizing hair dermal cells for allogenic transplants with reduced concern of rejection. In this manuscript we investigate the first, but fundamental steps in this approach, specifically: a) can human hair follicle dermal cells support growth of overlying epithelial cells in a three dimensional skin model, b) how does their capacity to support epidermal keratinocytes compare with interfollicular dermal fibroblasts and c) do they retain any follicle specific characteristics such as the ability to induce follicular structures?

\section{Materials and Methods}

\section{Establishment of cell cultures}

Occipital scalp skin samples were obtained as discarded tissue, after receiving an ethical approval, and Institutional Review Board exemption from Columbia University Medical Center. To isolate hair follicle cells, follicle endbulbs were transected, and then inverted using $27 \mathrm{G}$ needles, enabling microdissection of the dermal papilla and dermal sheath ${ }^{44}$. Papilla, or sheath were placed in $35 \mathrm{~mm}$ culture dishes containing $20 \%$ FBS (Invitrogen) in dMEM containing $4.5 \mathrm{mg} / \mathrm{ml}$ glucose (Invitrogen) with 1x Penicillin, Streptomycin, and Fungizone (Invitrogen). They were allowed to settle in an incubator for 10 days, after which time the papilla, or sheath structures had collapsed, and cells had started to migrate out from them in an explant, starburst formation. Medium was also changed to $10 \%$ FBS in dMEM with $1 \mathrm{x}$ Penicillin and Streptomycin. Once $35 \mathrm{~mm}$ dishes were filled with cells, they were passaged using $0.5 \%$ Trypsin-EDTA, and split at a 1:2 ratio. To isolate fibroblasts, skin biopsies were transected using a scalpel blade just beneath the epidermis, roughly separating the papillary and reticular dermis. Hair fibers were removed, and the papillary dermis was then chopped up into fine pieces using small scissors. These small pieces were adhered to a $35 \mathrm{~mm}$ dish, and once attached 20\% FBS in dMEM with 1x Penicillin, Streptomycin, and Fungizone was added to the

This article is protected by copyright. All rights reserved. 
culture. Fibroblasts cells migrated out of these explants, which were then passaged in a similar manner to the papilla and sheath cells. Fibroblasts, dermal sheath, and dermal papilla cell lines that were matched (from the same skin donor), were then used for comparison against each other in later experiments. Cells from three different donors were used in these experiments.

Keratinocytes were subsequently isolated from discarded neonatal skin. Neonatal skin samples were designated as nonhuman subject research under 45 CFR Part 46, and we therefore received an Institutional Review Board exemption at Columbia University to use these materials. Briefly, skin was placed in $2.4 \mathrm{mg} / \mathrm{ml}$ Dispase overnight at $4^{\circ} \mathrm{C}$, after which time the epidermis and dermis were separated using forceps. The epidermis was roughly minced, then placed into TrypLE® (Invitrogen) for 30 minutes at $37^{\circ} \mathrm{C}$, after which time the reaction was stopped, and cells were passed through a $70 \mu \mathrm{m}$ cell strainer. Isolated keratinocytes were grown in Epilife $($ (Invitrogen).

\section{Generation of in vitro bioengineered skin}

To establish skin constructs we followed the methods described in detail by Carlson et al., ${ }^{12}$, with only slight modifications. Instead of using bovine collagen from Organogenesis, we used $3.7 \mathrm{mg} / \mathrm{ml}$ rat tail collagen purchased from BD Biosciences. Moreover, 010 medium was established using dMEM powder (Sigma-Aldrich, D5030) rather than DME powder from JRH Biosciences.

Constructs were established in parallel from fibroblasts, dermal papilla cells and dermal sheath cells from matched donors. After establishing dermal scaffolds, constructs were left to contract for 7 days prior to the addition of keratinocytes. Keratinocytes used were at passage 1 or 2 grown in serum free medium. After adding keratinocytes, constructs were kept for 4 days in 12mls Epidermalization I medium, 2 days in Epidermalization II medium, prior to feeding with Cornification medium ${ }^{12}$. Only $7 \mathrm{mls}$ cornification medium was used, enabling exposure of the construct surface to the air liquid

This article is protected by copyright. All rights reserved. 
interface. Constructs were maintained in cornification medium for a further 11 days, meaning the total time between establishing the dermal layer and ending the experiment was 24 days.

\section{Generation of skin in vivo}

Dermal fibroblasts, dermal papilla cells, dermal sheath cells, or keratinocytes were trypsinized, counted and resuspended in PBS. Ten million keratinocytes were then combined with either ten million fibroblasts, or ten million dermal papilla cells. Cell mixtures were centrifuged, and resuspended in $100 \mu \mathrm{l}$ PBS. This resultant cell slurry comprising of epidermal and dermal cells was then pipetted into a hole located on the top of a sterile silicone chamber (Renner GmbH, Germany), which had previously been inserted under the dorsal skin of a SCID mouse. This work was performed after approval from the Institutional Animal Care and Use Committee at Columbia University. After one week the silicone chamber was removed, and cells were left for a further two weeks. Three weeks after cell grafting, skin structure could be observed. This was harvested for subsequent analysis.

\section{Histological and immunofluorescence analysis of bioengineered skin}

Skin constructs were placed in $2 \mathrm{M}$ sucrose overnight, prior to embedding in OCT compound, and freezing. $7 \mu \mathrm{M}$ sections were cut on a cryostat, and mounted onto positively charged glass slides. Sections were air-dried at room temperature for 1 hour, then fixed with either $4 \%$ paraformaldehyde in PBS for 10 minutes at room temperature, or with chilled acetone:methanol $1: 1$ at $-20^{\circ} \mathrm{C}$, for 7 minutes. Fixative was removed by washing slides in PBS 3 times, with 3 minute intervals at room temperature. 2\% Fish Skin Gelatin (Sigma) in PBS was applied to sections for 1 hour, used to block non-specific binding. Block was removed by tapping the edge of the slide gently, and primary

This article is protected by copyright. All rights reserved. 
antibodies (Table 1) were then applied to slides prior to them being left overnight at $4^{\circ} \mathrm{C}$. The next day, primary antibodies were removed by washing slides 3 times, with 3 minute intervals in PBS. Secondary antibodies (goat anti-rabbit 488/594, or goat anti-mouse 488/594, Molecular Probes) were diluted 1:800, and then applied to slides and left for 1 hour at room temperature, shielded from light. Secondary antibodies were washed off slides using three washes of PBS, and coverslips were mounted using vectashield containing DAPI (Vector labs). Slides were visualized, and images taken, on a LCM Excitor Confocal microscope.

For histological analysis sections were stained using haematoxylin and eosin, prior to mounting coverslips with DPX.

\section{Transmission electron microscopy analysis}

Skin constructs were placed in freshly made Karnovsky fixative, comprising of $2 \%$ paraformaldehyle and $2.5 \%$ Glutaraldehyde in $0.1 \mathrm{M}$ phosphate buffer ${ }^{45}$. Specimens were then postfixed in $1 \%$ OsO4 (Agar Scientific) buffered in 0.2M Sodium Phosphate buffer, pH 7.4. After post fixing, they were dehydrated through a series of ascending grades of ethanol $(70 \%, 95 \%)$ with 3 changes, each lasting 15 minutes followed by immersion in absolute ethanol, for three 30 minute intervals. Following this, the samples were immersed in an intermediate solution consisting of a 50:50 mix of $100 \%$ alcohol and propylene oxide. After 3 changes, 10 minutes apart, they were moved to propylene oxide, again with 3 changes, and 10 minute intervals. They were then placed in a fresh 50:50 araldite resin:propylene oxide mix and left overnight for the resin to infiltrate and the propylene oxide to evaporate. The resin was composed of (in 46.2 grams) 23 grams Araldite CY 212, 22 grams DDSA and 1.2 grams BDMA (Agar Scientific). The next day specimens were placed in a fresh resin, for 30 minutes, then into rubber molds with fresh araldite, which was then left to polymerize for 48 hours at $60^{\circ} \mathrm{C}$. Ultrathin sections were cut using a diamond knife on a Reichert

This article is protected by copyright. All rights reserved. 
Ultracut S Ultramicrotome and transferred to formvar coated grids. Sections were then stained with $1 \%$ Uranyl acetate in $70 \%$ ethanol, washed in water, then stained with Reynolds Lead Citrate for visualizing. Ultrathins were imaged on a Hitachi H7600 Transmission Electron Microscope.

\section{Results}

\section{Differentiation, stratification and proliferation in bioengineered skins}

After establishment of in vitro skin constructs the morphology of the newly formed epidermis was assessed on haematoxylin and eosin stained sections. At the 24 day time point when skin constructs were embedded, they had been in cornification medium, raised to the air liquid interface and undergoing a program of differentiation for 11 days. On each of the three dermal substrates, epidermal proliferation and differentiation had occurred to form a continuous epidermal covering. On top of all three dermal cell types, epidermal cells perpendicular to the dermal-epidermal junction formed a clear basal layer. (Fig. 1a-c). Above this, a noticeable change in cell orientation was visible, resulting in more horizontally aligned cells, forming the stratum spinosum. Above the spinous layer, the stratum granulosum layer was characterized by cells containing keratohyalin granules. The outer layer of the epidermis, the stratum corneum was also present in all skin constructs, as a thickened non-nucleated layer. There were no obvious differences in the equivalents containing hair follicle dermal cells, when compared to a fibroblast support layer, indicating that hair follicle dermal cells are capable of supporting growth and differentiation of overlying epidermal cells, in a manner similar to regular interfollicular fibroblasts.

Immunofluorescence was then used to assess whether the equivalents expressed specific differentiation markers, present within the various layers of normal epidermis. The epidermis has a changing profile of keratins that provide stability to the epidermal cells, but also enable

This article is protected by copyright. All rights reserved. 
discrimination of differentiated layers. Keratin $5(\mathrm{~K} 5)$ and keratin 1 (K1) were investigated as the former marks basal epidermis, while the latter demarcates keratins present within the suprabasal epidermis, not including the stratum corneum. Loricrin (LOR) is a major component of the cornified cell envelope barrier and is present within the terminally differentiated stratum corneum. While K5 was exclusive to the basal layer in control skin, within skin equivalents supported by all three dermal cell types $\mathrm{K} 5$ was seen both in the basal layer and at weaker levels in the suprabasal keratinocytes (Fig. 1d-g). Comparatively, in both control skin and all skin constructs, $\mathrm{K} 1$ was exclusively within the suprabasal keratinocytes and was absent from the basal layer (Fig. 1h-k). Moreover, LOR expression was restricted to the stratum corneum of control skin, and bioengineered skin constructs incorporating either fibroblasts, or hair follicle dermal cells, indicating that a normal program of differentiation and stratification was occurring in all cases (Fig. 11-o).

While the presence of cytokines within skin constructs has been shown to stimulate wound repair after skin construct grafting ${ }^{46}$, an additional important factor is the viability of the graft. When the presence of KI67 was assessed a low number of proliferating basal keratinocytes was observed in the control skin and similar labeling was seen within all three skin construct types, in addition to the occasional proliferating dermal cell (Fig. 1p-s).

Desmosomes are present throughout the basal and suprabasal epidermis, while corneodesmosomes are in the stratum corneum. Desmosomes enable connection of adjacent cells within the epidermis and provide integrity, preventing shearing of the skin. The expression of both desmoglein 3 (DSG3), and desmoplakin (DSP), was investigated to evaluate desmosomal protein synthesis within our bioengineered skins. In control skin, DSG3 was expressed within both the basal and suprabasal layers, with strongest expression observed in the stratum basale and stratum spinosum (Fig. 2a). DSP was expressed in all basal and suprabasal cells, although strongest expression was observed within the stratum spinosum and granulosum. Within skin constructs, with dermal fibroblasts, papilla, or sheath cells supporting the keratinocytes, DSG3 was observed in both

This article is protected by copyright. All rights reserved. 
the basal and suprabasal layers of skin, with the strongest expression seen in the basal layer (Fig. $2 b-d)$. DSP was observed at low levels in the basal layer, but increased in intensity within the stratum spinosum (Fig. 2e-h). Transmission electron microscopy showed that in constructs supported by all three cell types, numerous desmosomes were linking cells throughout the basal and suprabasal layers of the epidermis. (Fig. 2i-k).

\section{Basement membrane formation in bioengineered skins}

Immunofluorescence was used to assess the expression of basement membrane components within the bioengineered skin constructs. This is predominantly comprised of extracellular matrix proteins, synthesized by the epidermal keratinocytes. However, dermal cells are important here, as they stimulate the epidermis to deposit a basal lamina ${ }^{47}$. While levels of type VII collagen (COL7) staining were relatively similar in the basement membrane of different bioengineered constructs (Fig. 3a-d), type IV collagen (COL4) labeling was most intense in the basement membrane of constructs supported by dermal sheath cells from the hair follicle (Fig. 3e-h). Quantification of membrane thickness by assessing COL4 expression revealed that dermal sheath supported constructs had significantly thicker basement membranes compared to the fibroblast and dermal papilla constructs (Fig 3i).

The extent of basal lamina formation in skin constructs was then assessed using transmission electron microscopy. While some basal lamina could be detected in all three bioengineered skin constructs, within the fibroblast supported specimens the basal lamina was patchy, not showing continuity across the entire construct (Fig. 3j). Moreover, the basal lamina, which has a clear three layered structure in electron micrographs, was often missing its most basal layer of the three. Dermal papilla supported constructs had a largely consistent, three layered basal lamina, however, there were areas where it was not intact and resembled the basal lamina in the fibroblast supported

This article is protected by copyright. All rights reserved. 
constructs (Fig. 3k). Contrastingly, the basal lamina in hair follicle dermal sheath supported constructs was thickened, rarely showing areas of breakage, indicating a consistent deposition across the entire construct (Fig. 3l). In dermal sheath supported constructs, anchoring fibril-like projections were observed from the constructs that made contact with the basal lamina (Fig. S1). This was rarely observed within the constructs supported by either dermal fibroblasts, or dermal papilla cells.

\section{Dermal identity in bioengineered skins}

An interesting facet about hair follicles, is that the dermal sheath expresses alpha smooth muscle actin (aSMA), an actin isoform usually present in smooth muscle cells, or myofibroblasts ${ }^{48}$. In healing wounds, the transient presence of aSMA expressing myofibroblasts enables wound contracture to occur ${ }^{49,50}$. Using antibodies specific to aSMA, we analyzed the expression in the dermis of our three different bioengineered skin constructs (Fig. 4a-d). In control skin that is unwounded, aSMA is usually only located around blood vessels, and in hair follicle dermal sheath. However, in culture aSMA switches on in dermal papilla cells, so it is expressed by both hair follicle fibroblast populations ${ }^{48}$. We did not observe aSMA within the dermis of constructs supported by fibroblasts, however, high expression levels were seen in constructs supported by both hair follicle dermal sheath and hair follicle dermal papilla cells. This indicates that hair follicle dermal cells maintain their cell identity in engineered skin constructs.

In addition to differences in expression, hair follicle dermal cells are unique from interfollicular fibroblasts in a number of ways. In specific conditions, human dermal papilla cells have been shown to be capable of promoting hair follicle growth when combined with competent human epithelium or human epithelial cells ${ }^{42,51}$. However, within our timeframe in vitro we did not observe any induction of hair follicles, or anomalous follicle-like structures.

This article is protected by copyright. All rights reserved. 


\section{Formation of de novo skin in vivo}

In addition to analyzing engineered skins assembled layer by layer in vitro, we also assessed whether all three fibroblast populations could support establishment and organization of human keratinocytes in vivo. When we introduced a slurry of keratinocytes together with either fibroblasts, dermal papilla cells or dermal sheath cells, into chambers on the backs of mice, they quickly selforganized to form a skin structure. Histological analysis of these regions after 3 weeks showed that cells had reorganized with dermal cells on the inner surface, and keratinocytes exposed to the external air interface (Fig. S2a-c). Further analysis was only performed comparing hair follicle dermal papilla, and interfollicular fibroblasts. In epidermis supported by both fibroblasts, and dermal papilla cells, the keratinocytes had differentiated and formed stratified layers, creating a human skin on the back of a mouse (Fig. S2d-f). K1 was expressed in the suprabasal layers of skins supported with both fibroblasts and dermal papilla cells, while DSG3 was clearly present within the basal and spinous layers (Fig. S3a-d). Basement membranes were also established, as indicated by the presence of COL7 (Fig. S4a-b) and COL4 (Fig. S4c-d) in the in vivo skins supported by both fibroblasts and dermal papilla cells. Additionally, while in vitro constructs are established in a type I collagen (COL1) scaffold, in vivo the dermal cells have to produce the COL1 themselves. COL1 deposition was observed in skins established using both fibroblasts and dermal papilla cells (Fig. S4e-f). Lastly, while skins established with dermal papilla cells did retain some hair follicle characteristics, by expressing aSMA within the dermis, there was a noticeable absence of hair follicle structures in any skins established in vivo at the 3 week time point when the experiment was completed.

This article is protected by copyright. All rights reserved. 


\section{Discussion}

In this study our principal goal was to determine if human hair follicle derived dermal cells were capable of supporting the growth of epithelial cells in a three dimensional construct to form a viable bioengineered skin. We have successfully demonstrated that both dermal papilla, and dermal sheath cells can replace interfollicular fibroblasts in skin constructs, and, in one specific feature, basement membrane formation, dermal sheath cells are superior to fibroblasts. This supports the idea that hair follicle dermal cells have a dual capacity in the skin. In addition to being integral to the hair follicle, and driving hair follicle cycling, they are also capable of switching to support epidermal growth and renewal in a regenerative context ${ }^{52}$. In this regard they parallel to some extent the follicle epithelial outer root sheath progenitors, which can also display a dichotomy of activity within skin. Indeed human hair follicle epithelial cells have already been used for skin replacement in a clinical context ${ }^{40}$. We also show that follicle dermal cells are capable of constituting skin dermis as part of reconstituted skin dermis in vivo, but follicle dermal cells did not induce follicular structures either in vitro or in vivo.

One predominant requirement of the dermal portion of a bioengineered skin is to support the overlying epidermis, and maintain the cells in a viable state. The dermis and epidermis have a synergistic relationship, perhaps explaining why bioengineered skins with only an epidermis are not long lived. The dermis provides structural support for the overlying keratinocytes, enabling production of key basement membrane components. Interestingly, one area where the hair follicle dermal cells appeared superior to interfollicular fibroblasts was in their capacity to influence the establishment and maintenance of a basal lamina. Dermal cells are capable of stimulating overlying keratinocytes to deposit a basal lamina ${ }^{53}$. In our skin constructs containing hair follicle dermal cells, in particular dermal sheath, we saw an increase in COL4 expression compared to fibroblast supported constructs, coinciding with a formation of a robust and uniform basal lamina as shown by electron microscopy. This capacity to stimulate basal lamina formation is perhaps not surprising,

This article is protected by copyright. All rights reserved. 
given that in situ the dermal sheath contributes to and stimulates the formation of, the substantial and complex basement membrane structure termed the glassy membrane, which separates dermal sheath from the epithelium around the hair follicle.

One key role of a bioengineered skin is to promote wound closure after grafting, by providing a moist wound environment which will stimulate skin repair and replacement. Skin constructs supported by hair follicle dermal cells had a well-stratified epidermis and cornified stratum corneum layer as indicated by LOR staining. This will enable the bioengineered skin to form a barrier to external influences, while also trapping in water and solutes to create an environment that will promote healing. Another key role of bioengineered skins is to produce cytokines that positively influence the healing process. Interestingly, VEGF is expressed at very high levels within hair follicle dermal cells ${ }^{33}$, allowing us to postulate that grafted skin constructs containing hair follicle dermal cells may in some way promote vascularization, and therefore integration of the skin construct to enable faster wound healing.

One interesting feature of our hair follicle derived skin constructs was the presence of aSMA, a marker of myofibroblasts, within the dermis of skin constructs. This demonstrated that the hair follicle derived cells, while assuming the role of dermal fibroblasts were, nevertheless, maintaining some follicle specific characteristics. Hair follicle dermal sheath express aSMA in vivo, while both dermal papilla and dermal sheath express the marker in vitro ${ }^{48}$. There is a positive correlation with aSMA expression, and the contractile activity of fibroblasts ${ }^{54}$. We therefore hypothesize that the presence of aSMA within grafted skin constructs would aid to create an environment conducive to healing, by promoting contracture of the wound.

We have previously shown in rodents that adult whisker follicle dermal cells can contribute to wounded skin dermis and form the dermis in a reconstructed skin model in vivo ${ }^{55}$. Here, we have demonstrated that likewise, human hair follicle dermal cells are capable of supporting overlying

This article is protected by copyright. All rights reserved. 
epidermal cells in a bioengineered skin. While several groups are exploring the possibility of incorporating alternative cell types into skin constructs, it is still essential that we investigate alternative cell types within the skin as a novel cell source for engineering skin.

Our findings within this manuscript support our previous observations that hair follicle dermal cells are superior to fibroblasts when used as a feeder layer to support keratinocytes in culture ${ }^{35}$. With the recent explosion of interest in de novo hair follicle formation, we have to postulate if incorporation of hair follicle dermal cells within skin constructs will be a stepping stone to enable growth of hairy bioengineered skins. Our bioengineered skins were maintained for 3-4 weeks in culture, and at no point in time did we observe de novo follicle formation within our constructs. Similarly, we did not observe any signs of hair follicle formation in our in vivo skins. Others have produced follicles in their skin constructs ${ }^{42,56}$ raising the question of why this did not occur here? This could be due to different methods; in other protocols in vitro skin constructs were established then grafted onto nude mice to ensure survival up to 15 weeks, while ours were only maintained for 3-4 weeks ${ }^{42}$. Alternatively, incorporating dermal papilla spheroids into the skin construct dermis can enable papilla inductivity ${ }^{56}$, while in our study we only used dispersed hair follicle dermal cells. Another possibility is that there are individual and/or site specific differences between the hair follicle populations used in different studies ${ }^{42}$. Notwithstanding, incorporation of hair follicles into constructs by exploiting the inherent properties of the dermal cells to induce new hair structures is a first step to recreating a functional skin, however, controlling follicle depth and directionality are other obstacles which lie ahead ${ }^{57}$. Current bioengineered human skins are devoid of organized hair follicles, and indeed other structures that such as blood vessels and nerves, which together make a the skin a highly complex structure. Introduction of various skin derived cell types into bioengineered constructs, will no doubt, over time, enable the creation of bioengineered skins that more closely recreate or mimic the complexity of human skin.

This article is protected by copyright. All rights reserved. 


\section{Acknowledgements}

We are grateful to Dr. Mark Carlson for sharing his expertise on bioengineered skin generation. We also thank Dr. Robert Bernstein for generously providing us with human skin for dermal cell isolation in this study, Dr. Jim Wahl for gifting us the DSG3 and DSP antibodies, and Benjamin Solis-Cohen and Christine Richardson for technical assistance. This work was supported by funding from the Dermatology Foundation to $\mathrm{CAH}$, the Medical Research Council (G1000846) and British Skin Foundation to CABJ, and by a Core facilities grant to the Skin Disease Research Center at Columbia University (P30AR044535 from NIH/NIAMS).

\section{References}

1. Chuong CM, Nickoloff BJ, Elias PM, et al. What is the 'true' function of skin? Exp Dermatol 2002; 11(2):159-87.

2. Loots MA, Lamme EN, Zeegelaar J, et al. Differences in cellular infiltrate and extracellular matrix of chronic diabetic and venous ulcers versus acute wounds. $J$ Invest Dermatol 1998; 111(5):850-7.

3. Guo S, Dipietro LA. Factors affecting wound healing. J Dent Res 2010; 89(3):219-29.

4. Gottrup F, Apelqvist J, Price P. Outcomes in controlled and comparative studies on nonhealing wounds: recommendations to improve the quality of evidence in wound management. $J$ Wound Care 2010; 19(6):237-68.

5. McHeik JN, Barrault C, Levard G, et al. Epidermal healing in burns: autologous keratinocyte transplantation as a standard procedure: update and perspective. Plast Reconstr Surg Glob Open 2014; 2(9):e218.

6. Uitto J, Christiano AM, McLean WH, et al. Novel molecular therapies for heritable skin disorders. J Invest Dermatol 2012; 132(3 Pt 2):820-8.

7. MacNeil S. Progress and opportunities for tissue-engineered skin. Nature 2007; 445(7130):874-80.

8. Huang S, Fu X. Tissue-engineered skin: bottleneck or breakthrough. Int J Burns Trauma 2011; 1(1):1-10.

9. Rheinwald JG, Green H. Serial cultivation of strains of human epidermal keratinocytes: the formation of keratinizing colonies from single cells. Cell 1975; 6(3):331-43.

This article is protected by copyright. All rights reserved. 
10. Wright KA, Nadire KB, Busto $P$, et al. Alternative delivery of keratinocytes using a polyurethane membrane and the implications for its use in the treatment of full-thickness burn injury. Burns 1998; 24(1):7-17.

11. Zaulyanov L, Kirsner RS. A review of a bi-layered living cell treatment (Apligraf) in the treatment of venous leg ulcers and diabetic foot ulcers. Clin Interv Aging 2007; 2(1):93-8.

12. Carlson MW, Alt-Holland A, Egles $\mathrm{C}$, et al. Three-dimensional tissue models of normal and diseased skin. Curr Protoc Cell Biol 2008; 41:19.9.1-.9.7.

13. Gangatirkar P, Paquet-Fifield S, Li A, et al. Establishment of 3D organotypic cultures using human neonatal epidermal cells. Nat Protoc 2007; 2(1):178-86.

14. El Ghalbzouri A, Commandeur S, Rietveld MH, et al. Replacement of animal-derived collagen matrix by human fibroblast-derived dermal matrix for human skin equivalent products. Biomaterials 2009; 30(1):71-8.

15. Orgill DP, Butler C, Regan JF, et al. Vascularized collagen-glycosaminoglycan matrix provides a dermal substrate and improves take of cultured epithelial autografts. Plast Reconstr Surg 1998; 102(2):423-9.

16. Chan RK, Zamora DO, Wrice NL, et al. Development of a vascularized skin construct using adipose-derived stem cells from debrided burned skin. Stem Cells Int 2012; 2012:Article ID:841203.

17. Schlabe J, Johnen C, Schwartlander R, et al. Isolation and culture of different epidermal and dermal cell types from human scalp suitable for the development of a therapeutical cell spray. Burns 2008; 34(3):376-84.

18. Liu P, Deng Z, Han S, et al. Tissue-engineered skin containing mesenchymal stem cells improves burn wounds. Artificial organs 2008; 32(12):925-31.

19. Collawn SS, Banerjee NS, de la Torre J, et al. Adipose-derived stromal cells accelerate wound healing in an organotypic raft culture model. Annals of plastic surgery 2012; 68(5):501-4.

20. Li H, Chu Y, Zhang Z, et al. Construction of bilayered tissue-engineered skin with human amniotic mesenchymal cells and human amniotic epithelial cells. Artificial organs 2012; 36(10):911-9.

21. Mine S, Fortunel NO, Pageon $\mathrm{H}$, et al. Aging alters functionally human dermal papillary fibroblasts but not reticular fibroblasts: a new view of skin morphogenesis and aging. PLoS One 2008; 3(12):e4066.

22. Shamis $\mathrm{Y}$, Hewitt KJ, Carlson MW, et al. Fibroblasts derived from human embryonic stem cells direct development and repair of 3D human skin equivalents. Stem cell research \& therapy 2011; 2(1):10.

23. Driskell RR, Lichtenberger BM, Hoste E, et al. Distinct fibroblast lineages determine dermal architecture in skin development and repair. Nature 2013; 504(7479):277-81.

This article is protected by copyright. All rights reserved. 
24. Richardson GD, Arnott EC, Whitehouse CJ, et al. Plasticity of rodent and human hair follicle dermal cells: implications for cell therapy and tissue engineering. The journal of investigative dermatology Symposium proceedings / the Society for Investigative Dermatology, Inc [and] European Society for Dermatological Research 2005; 10(3):180-3.

25. Bin S, Li HD, Xu YB, et al. BMP-7 attenuates TGF-beta1-induced fibroblast-like differentiation of rat dermal papilla cells. Wound Repair Regen 2013; 21(2):275-81.

26. Hou-dong L, Bin S, Ying-bin X, et al. Differentiation of rat dermal papilla cells into fibroblast-like cells induced by transforming growth factor beta1. Journal of cutaneous medicine and surgery 2012; 16(6):400-6.

27. Jahoda CA, Reynolds AJ. Hair follicle dermal sheath cells: unsung participants in wound healing. Lancet 2001; 358(9291):1445-8.

28. Ansell DM, Kloepper JE, Thomason HA, et al. Exploring the "hair growth-wound healing connection": anagen phase promotes wound re-epithelialization. J Invest Dermatol 2011; 131(2):518-28.

29. Zawacki BE, Jones RJ. Standard depth burns in the rat: the importance of the hair growth cycle. Br J Plast Surg 1967; 20(4):347-54.

30. Jimenez F, Garde C, Poblet E, et al. A pilot clinical study of hair grafting in chronic leg ulcers. Wound Repair Regen 2012; 20(6):806-14.

31. Yang Z, Liu J, Zhu N, et al. Comparison between hair follicles and split-thickness skin grafts in cutaneous wound repair. Int J Clin Exp Med 2015; 8(9):15822-7.

32. Jimenez F, Poblet E, Izeta A. Reflections on how wound healing-promoting effects of the hair follicle can be translated into clinical practice. Exp Dermatol 2015; 24(2):91-4.

33. Kozlowska U, Blume-Peytavi U, Kodelja V, et al. Expression of vascular endothelial growth factor (VEGF) in various compartments of the human hair follicle. Arch Dermatol Res 1998; 290(12):661-8.

34. Bassino E, Gasparri F, Giannini V, et al. Paracrine crosstalk between human hair follicle dermal papilla cells and microvascular endothelial cells. Exp Dermatol 2015; 24(5):388-90.

35. Hill RP, Gardner A, Crawford HC, et al. Human hair follicle dermal sheath and papilla cells support keratinocyte growth in monolayer co-culture. Exp Dermatol 2013.

36. Qi SH, Liu P, Xie JL, et al. Experimental study on repairing of nude mice skin defects with composite skin consisting of xenogeneic dermis and epidermal stem cells and hair follicle dermal papilla cells. Burns 2008; 34(3):385-92.

37. Lenoir MC, Bernard BA, Pautrat G, et al. Outer root sheath cells of human hair follicle are able to regenerate a fully differentiated epidermis in vitro. Dev Biol 1988; 130(2):610-20.

38. Hoeller D, Huppertz B, Roos TC, et al. An improved and rapid method to construct skin equivalents from human hair follicles and fibroblasts. Exp Dermatol 2001; 10(4):264-71.

This article is protected by copyright. All rights reserved. 
39. Kobayashi T, Enomoto K, Wang YH, et al. Epidermal structure created by canine hair follicle keratinocytes enriched with bulge cells in a three-dimensional skin equivalent model in vitro: implications for regenerative therapy of canine epidermis. Vet Dermatol 2013; 24(1):77-83 e19-20.

40. Limat A, Mauri D, Hunziker T. Successful treatment of chronic leg ulcers with epidermal equivalents generated from cultured autologous outer root sheath cells. J Invest Dermatol 1996; 107(1):128-35.

41. Sriwiriyanont $P$, Lynch KA, Maier EA, et al. Morphogenesis of chimeric hair follicles in engineered skin substitutes with human keratinocytes and murine dermal papilla cells. Exp Dermatol 2012; 21(10):783-5.

42. Thangapazham RL, Klover P, Wang JA, et al. Dissociated human dermal papilla cells induce hair follicle neogenesis in grafted dermal-epidermal composites. J Invest Dermatol 2014; 134(2):538-40.

43. Reynolds AJ, Lawrence C, Cserhalmi-Friedman PB, et al. Trans-gender induction of hair follicles. Nature 1999; 402(6757):33-4.

44. Gledhill K, Gardner A, Jahoda CA. Isolation and establishment of hair follicle dermal papilla cell cultures. Methods Mol Biol 2013; 989:285-92.

45. Karnovsky MJ. A formaldehyde-gluteraldehyde fixative of high osmolarity for use in electron microscopy. J Cell Biol 1965; 27:137-8A.

46. Mansbridge J, Liu K, Patch R, et al. Three-dimensional fibroblast culture implant for the treatment of diabetic foot ulcers: metabolic activity and therapeutic range. Tissue Eng 1998; 4(4):403-14.

47. Woodley $\mathrm{D}$, Regnier M, Prunieras $\mathrm{M}$. In vitro basal lamina formation may require nonepidermal cell living substrate. Br J Dermatol 1980; 103(4):397-404.

48. Jahoda CA, Reynolds AJ, Chaponnier C, et al. Smooth muscle alpha-actin is a marker for hair follicle dermis in vivo and in vitro. J Cell Sci 1991; 99 ( Pt 3):627-36.

49. Gabbiani G. The myofibroblast in wound healing and fibrocontractive diseases. J Pathol 2003; 200(4):500-3.

50. Tomasek JJ, Gabbiani G, Hinz B, et al. Myofibroblasts and mechano-regulation of connective tissue remodelling. Nature reviews Molecular cell biology 2002; 3(5):349-63.

51. Higgins CA, Chen JC, Cerise JE, et al. Microenvironmental reprogramming by threedimensional culture enables dermal papilla cells to induce de novo human hair-follicle growth. Proc Natl Acad Sci U S A 2013; 110(49):19679-88.

52. Jahoda CA. Cell movement in the hair follicle dermis - more than a two-way street? $J$ Invest Dermatol 2003; 121(6):ix-xi.

53. Werner S, Krieg T, Smola H. Keratinocyte-fibroblast interactions in wound healing. $J$ Invest Dermatol 2007; 127(5):998-1008.

This article is protected by copyright. All rights reserved. 
54. Hinz B, Celetta G, Tomasek JJ, et al. Alpha-smooth muscle actin expression upregulates fibroblast contractile activity. Mol Biol Cell 2001; 12(9):2730-41.

55. Gharzi A, Reynolds AJ, Jahoda CA. Plasticity of hair follicle dermal cells in wound healing and induction. Exp Dermatol 2003; 12(2):126-36.

56. Thangapazham RL, Klover $\mathrm{P}$, Li S, et al. A model system to analyse the ability of human keratinocytes to form hair follicles. Exp Dermatol 2014; 23(6):443-6.

57. Sriwiriyanont $P$, Lynch KA, McFarland KL, et al. Characterization of hair follicle development in engineered skin substitutes. PLoS One 2013; 8(6):e65664.

\begin{tabular}{|l|l|l|l|}
\hline Antigen & Source & $\begin{array}{l}\text { Species (raised } \\
\text { in) }\end{array}$ & Dilution used \\
\hline K5 & Covance & Rabbit & $1: 500$ \\
\hline K1 & Covance & Rabbit & $1: 500$ \\
\hline LOR & Covance & Rabbit & $1: 500$ \\
\hline DSG3 (Clone & Gift from J. Wahl & Mouse & $1: 3$ \\
\hline DSP (Clone & Gift from J. Wahl & Mouse & $1: 3$ \\
\hline 20B6) & & & \\
\hline COL7 & & Migma & $1: 500$ \\
\hline COL4 & Chemicon & Mouse & $1: 500$ \\
\hline COL1 & Chemicon & Rabbit & $1: 100$ \\
\hline
\end{tabular}

This article is protected by copyright. All rights reserved. 


\begin{tabular}{|l|l|l|l|}
\hline $\begin{array}{l}\text { aSMA (Clone } \\
1 \mathrm{~A} 4)\end{array}$ & DAKO & Mouse & $1: 30$ \\
\hline KI67 & Abcam & Rabbit & $1: 1000$ \\
\hline $\begin{array}{l}\text { Human Nuclei } \\
\text { (Clone 235-1) }\end{array}$ & Millipore & Mouse & $1: 100$ \\
\hline
\end{tabular}

Table 1. Primary antibodies used in this study.

\section{Figure legends}

Figure 1. Differentiation and proliferation in skin constructs. (a) H\&E staining of skin construct with fibroblasts in dermal support layer, compared to dermal papilla cells (b), or dermal sheath cells (c). Layers of the epidermis; stratum basale (SB), stratum spinosum (SS), stratum granulosum (SG), and stratum corneum (SC) can be seen in each construct. (d) K5 staining in normal human skin. In constructs supported by fibroblasts (e), dermal papilla cells (f), and dermal sheath cells (g), $\mathrm{K} 5$ is observed in the basal layer of the epidermis. (h) $\mathrm{K} 1$ staining in normal human skin. In constructs supported by fibroblasts (i), dermal papilla cells (j), and dermal sheath cells ( $\mathrm{k}), \mathrm{K} 1$ is observed in the suprabasal layers of the epidermis. (I) LOR staining in normal human skin. In constructs supported by fibroblasts $(m)$, dermal papilla cells $(n)$, and dermal sheath cells (o), LOR is observed in the stratum corneum. (p) Few basal keratinocytes are KI67 positive in normal human skin. Moreover, we see a similar expression pattern in basal keratinocytes supported by fibroblasts (q), dermal papilla cells ( $r$ ), and dermal sheath cells (s).

This article is protected by copyright. All rights reserved. 
Figure 2. Desmosomal junctions in skin constructs. DSG3 expression in normal human skin (a) is observed within the basal and spinous layers of the epidermis. In skin constructs supported by fibroblasts (b), dermal papilla cells (c), and dermal sheath cells (d), DSG3 expression is observed within the lower layers of the epidermis, however, the pattern is not as well defined as in control skin. (e) In control skin, DSP expression is observed in the suprabasal layers of the epidermis, in a defined cell border pattern. In epidermis supported by fibroblasts (f), dermal papilla cells (g), and dermal sheath cells (h), expression is observed in the suprabasal layers of the construct. Using transmission electron microscopy to visualize fibroblast supported constructs (i), in addition to those supported by dermal papilla (j), or dermal sheath (k) cells, we observed several desmosomes present at the cell surface of keratinocytes, enabling cell-cell contact and adhesion. Scale bars: $10 \mu \mathrm{m}$.

Figure 3. Basal lamina formation in skin constructs. (a) Within normal human skin, anchoring fibrils composed of COL7 are observed extending from the basal lamina into underlying collagen. In skin constructs with a dermal fibroblast (b), dermal papilla cell (c), or dermal sheath cell (d) support layer, COL7 is also observed, and nicely demarcates the boundary between the dermis and epidermis. (e) In normal human skin, the basal lamina is composed of COL4. Likewise, expression is observed in skin constructs with a fibroblast support (f), and a dermal papilla cell (g) support. In constructs supported by dermal sheath cells (h), high levels of collagen 4 were observed. (i) Whisker box plot of COL4 thickness, showing DS constructs had significantly thicker membranes than DP and DFi skins. (j) Transmission electron microscopy of the basal lamina (between arrows) of fibroblast supported constructs showed a thin lamella, often missing the most basal layer, and strewn with gaps. In constructs supported by dermal papilla cells (k), the lamina (between arrows) was thicker, but there were often areas where it failed to fully form. However, in skin constructs supported by dermal sheath cells (I), the basal lamina (between arrows) was nicely formed, and showed its

This article is protected by copyright. All rights reserved. 
characteristic three layered pattern. Moreover, the lamina was consistently visible across the entire construct. Scale bars: $1 \mu \mathrm{m} .{ }^{\star \star *} \mathrm{p}<0.001$.

Figure 4. Hair follicle identity in skin constructs. (a) In unwounded human skin, aSMA is usually only expressed in blood vessels, and in the dermal sheath of hair follicles. In the dermis of skin constructs supported by fibroblast cells (b), we observe very little aSMA. Comparatively, in the dermis of constructs supported by both dermal papilla cells (c), and dermal sheath cells (d), aSMA is readily observed, produced by cells within the collagen scaffold.
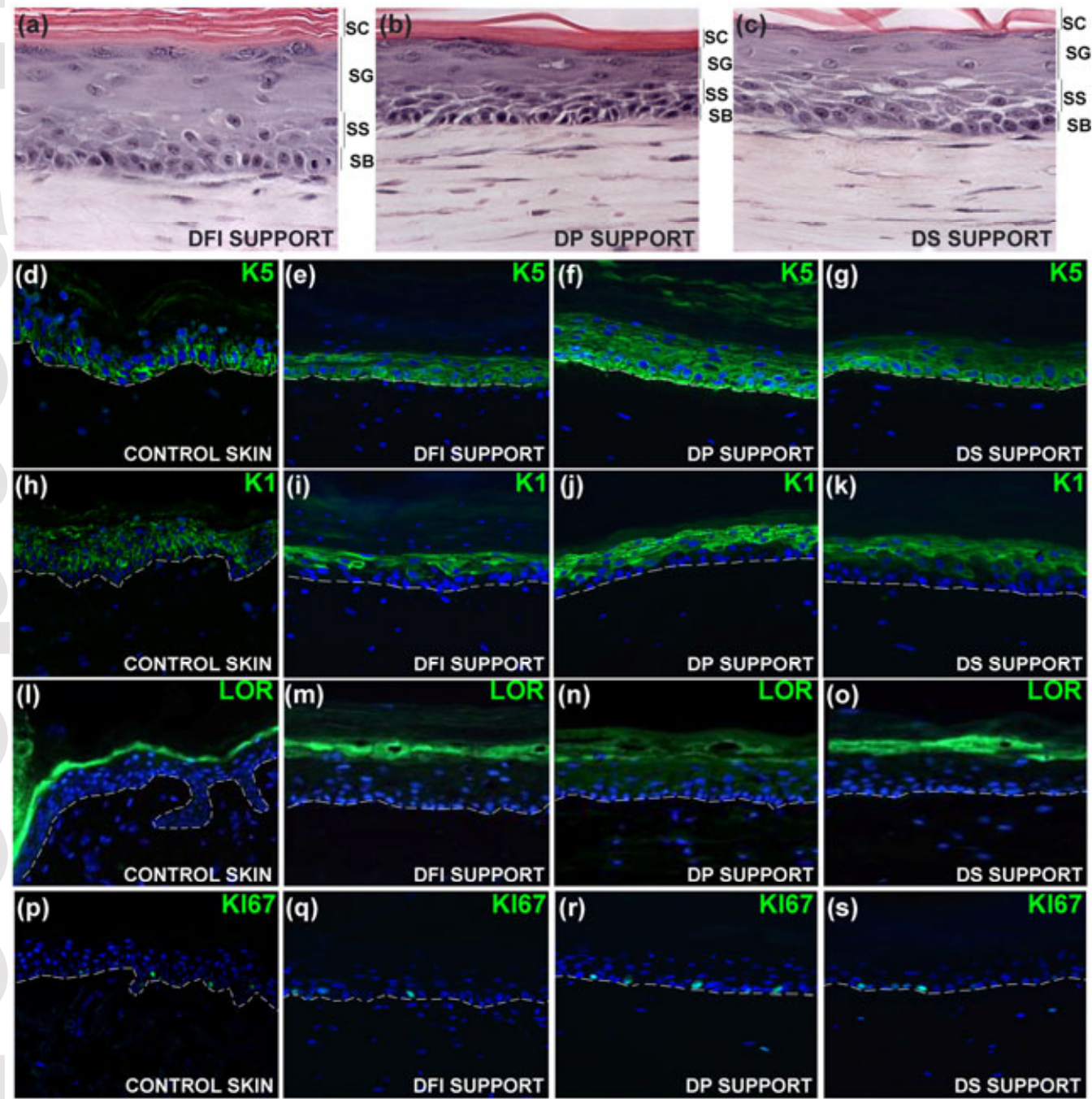

This article is protected by copyright. All rights reserved. 

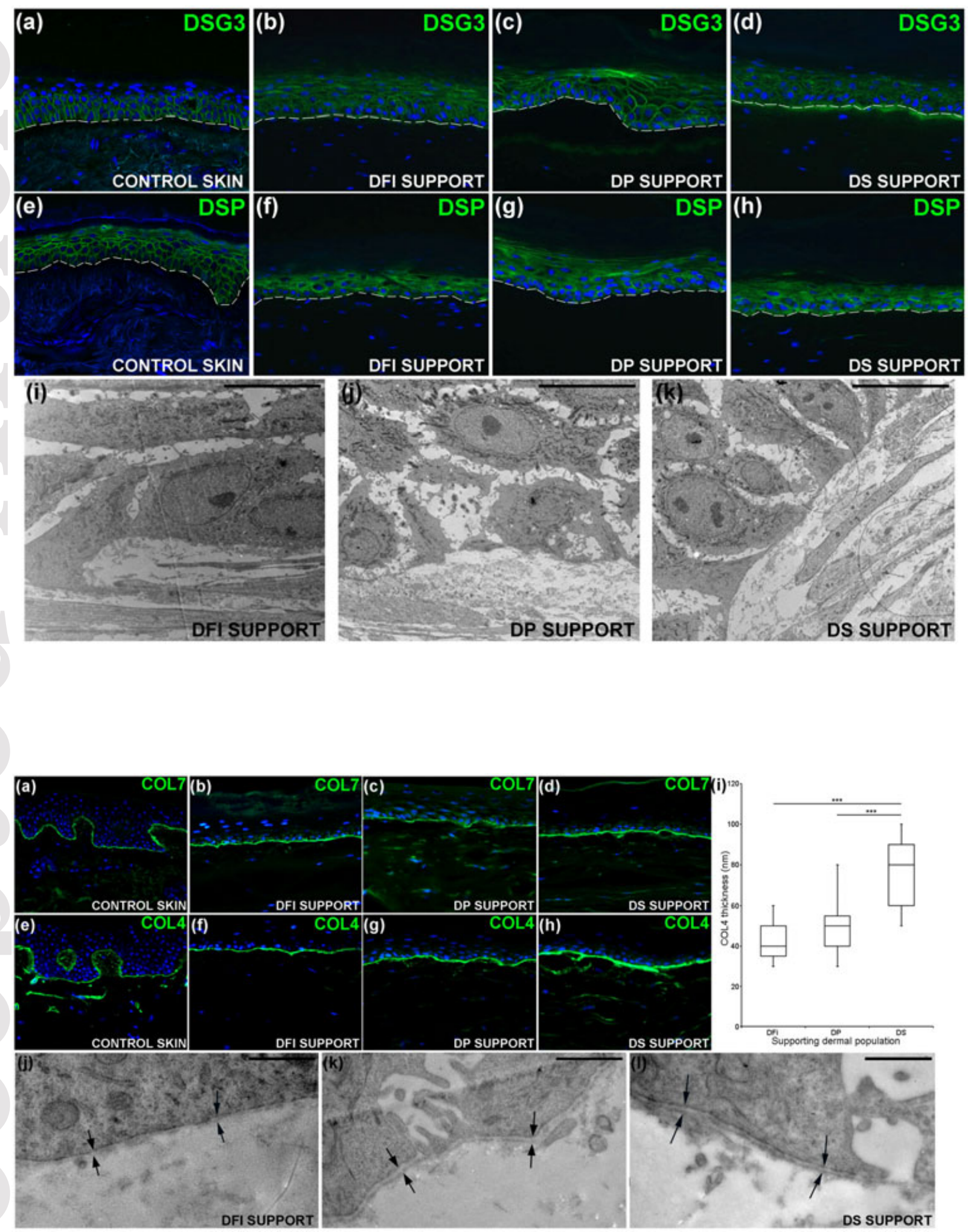

This article is protected by copyright. All rights reserved. 


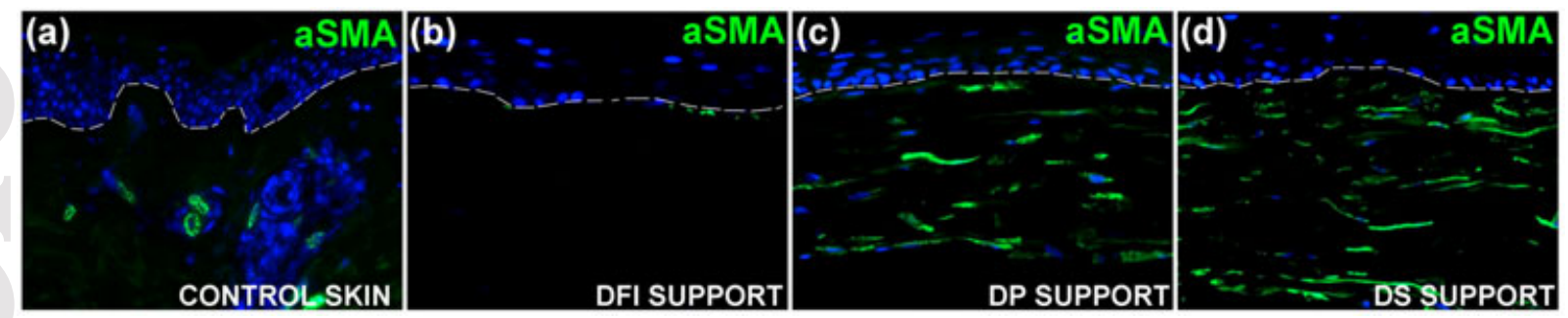

This article is protected by copyright. All rights reserved. 